\title{
ALGORITHM AND APPLICATION OF GCP-INDEPENDENT BLOCK ADJUSTMENT FOR SUPER LARGE-SCALE DOMESTIC HIGH RESOLUTION OPTICAL SATELLITE IMAGERY
}

\author{
Y. S. Sun ${ }^{1 *}$, L. Zhang ${ }^{1}$, B. Xu' ${ }^{1}$, Y. ZHANG ${ }^{2}$ \\ ${ }^{1}$ Chinese Academy Surveying \& Mapping, Beijing 100830 \\ ${ }^{2}$ School of Remote Sensing and Information Engineering, Wuhan University, Wuhan 430079
}

KEY WORDS: Rational Function Model (RFM); Virtual Control Points; Least-squares Block Adjustment; Alternating Direction Method; Geometric Constraints of Horizontal and Vertical

\begin{abstract}
:
1

The accurate positioning of optical satellite image without control is the precondition for remote sensing application and small/medium scale mapping in large abroad areas or with large-scale images. In this paper, aiming at the geometric features of optical satellite image, based on a widely used optimization method of constraint problem which is called Alternating Direction Method of Multipliers (ADMM) and RFM least-squares block adjustment, we propose a GCP independent block adjustment method for the large-scale domestic high resolution optical satellite image - GISIBA (GCP-Independent Satellite Imagery Block Adjustment), which is easy to parallelize and highly efficient. In this method, the virtual "average" control points are built to solve the rank defect problem and qualitative and quantitative analysis in block adjustment without control. The test results prove that the horizontal and vertical accuracy of multi-covered and multi-temporal satellite images are better than $10 \mathrm{~m}$ and $6 \mathrm{~m}$. Meanwhile the mosaic problem of the adjacent areas in large area DOM production can be solved if the public geographic information data is introduced as horizontal and vertical constraints in the block adjustment process. Finally, through the experiments by using GF-1 and ZY-3 satellite images over several typical test areas, the reliability, accuracy and performance of our developed procedure will be presented and studied in this paper.
\end{abstract}

\section{INTRODUCTION}

In recent years, the resolution of space and radiation of domestic satellite has been improved significantly. Through the application and research of survey satellite, such as "TH1 " "ZY-3" series satellites, many national industries have a deep understanding to the requirement and research of survey satellite, that lead to the great improvement in sensor design, manufacture and geometric calibration. However, the imaging system usually use the linear CCD imaging technology, the long focal length and narrow field angle result in the geometrical model having the characteristics of $3 \mathrm{~d}$ parallel projection, even after the rigorous on-orbit geometry calibration process, the satellite images will still have residual system errors caused by the drift error of satellite-borne GPS/ IMU, asynchrony between the pose and trace of satellite. In order to meet the needs of remote sensing monitoring and mapping application, the overall block adjustment technique is still needed. Therefore, satellite images-based accurate positioning without ground control point information is the precondition for obtaining global geographic and resource environmental information and monitoring changes in global resource environment.

When carrying the high-precision and GCP-independent positioning of domestic high-resolution satellite images, due to the lack of control point constraints, using the parameters which need adjustment as the free unknown parameter would lead to the morbid (rank loss) of the normal equation matrix, that will result in the instability of the adjustment accuracy and the distortion of the triangulation caused by the error excessive accumulation. Many researches about the GCP-independent block adjustment have been carried out. One kind of these methods is to make an on-orbit geometry calibration to obtain the accuracy (about $50 \mathrm{~m}$ ) of the ground positioning having the same order of magnitude. Then, based on the sensor imaging features, a specific follow-up block adjustment model is selected to carry out the overall block adjustment ${ }^{[1]-[5]}$. Or using the initial RPC model of image needing adjustment to generate a uniform distributed virtual control points, and then using these virtual control points as weighted observations, introducing them into the adjustment model to carry out the block adjustment in the area without ground control ${ }^{[6]-[8]}$. Another kind of methods is to carry out "GCP-independent" block adjustment by introducing multi-source control information (SAR image, high precision optical satellite image) ${ }^{[9]-[10]}$. Or using the model-independent method, the minimum elevation difference (LZD) method directly to carry out image matching between the image to be processed and the automatic control benchmark of the geographic information data to obtain a certain number of so-called "control points", then using these points to have a GCP-independent positioning of satellite image. In term of the accuracy of the imagery GCPindependent positioning, the "ZY-3" satellite image has been improved to $15 \mathrm{~m}$ after the calibration and the internal precision is better than 1 pixel, after the overall block adjustment without GCP, the plane and elevation accuracy of the image can be further increased to $5 \mathrm{~m}$ (medium error) ${ }^{[1]}$ [2][6][7][8]; the measured results of the "TH" satellite center show that, the root mean square error (RMSE) of "TH-1" satellite 01 was $10.3 \mathrm{~m} / 5.7 \mathrm{~m}$ (plane/elevation) after seven calibrations in China, and the accuracy of the satellite 02 was equal to the accuracy of satellite $01^{[3][4]}$. After the use of two domestic geometric calibration fields, the accuracy of the GCPindependent positioning of "TH-1" satellite NO.3 can achieved 7.2m /2.6m (plane/elevation) ${ }^{[5]}$.

Above all, the GCP-independent block adjustment of satellite images can be considered as, in the case of no ground control points, the overall block adjustment of the multi-source optical satellite imagery with the specific constraints (distance and Angle, etc.). Most GCP-independent adjustment used a kind of virtual control points, but the precision of these virtual control points is low (varied system error) and the precision is not consistent in the measurement area, so the GCPindependent adjustment is a kind of block adjustment under 
different precision control, the law of its error propagation is more complex, gross error detection and positioning are more difficult. Meanwhile, many experiments showed that the size and direction of the system error on the different points are almost consistent, but for the images of the same coverage area obtained on different orbit and time in a long-time interval, the size and direction of the system error are different, that shows a certain randomness, and even it can be partially offset each other.

Aiming at the above problems, this paper presents a "average" virtual control point based stereoscopic GCP-independent block adjustment method for large-scale domestic satellite image -GISIBA (GCP-Independent Satellite Imagery Block Adjustment). Based on the automatic and reliable acquisition of uniform distributed image tie points, the method comprehensively uses the "Alternating Direction Method" introduced from aerial photogrammetry and the total least squares adjustment algorithm to realize the total combined block adjustment of satellite images. First, the "Alternating Direction Method" is used to solve the unknowns' initial value, automatic detection and elimination of above medium scale gross error based on the parallel processing platform, and then according to the solving results, giving all unknowns a priori weights. Then, using the least square method, to solve the super-large-scale reformation normal equation, and the orientation parameters with high precision are obtained, which meets the requirement of the production of high precision image products. Carrying out block adjustment by constructing a virtual "average" control points, it addressed the "rank" problems in the GCP-independent adjustment, improved the state of normal equation of block adjustment system which benefits the stability and fast convergence of block; At the same time, it is convenient to analyze the relationship among the data coverage, imaging time interval and the satellite image GCP-Independent block adjustment. To solve the practical problems such as image mosaic in production, third-party geospatial data (such as SRTM DEM and Google Earth DOM) were introduced into the adjustment model as geometric constraints of horizontal and vertical. The accumulation of residual errors can be better reduced to obtain more reliable "unbiased" block adjustment results at final processing. In addition, to ensure the efficiency of block adjustment, parallel processing based on the OMP parallel method is used to realize the parallel processing of the "alternating direction method" and the multi-thread parallel computing based on the least-squares adjustment. Finally, through the experiments on ZY-3, GF-1 satellite images over several typical test areas, the reliability, accuracy and performance of our method are verified and studied in this paper.

\section{RFM-BASED SATELLITE IMAGE BLOCK ADJUSTMENT}

RFM based block adjustment model can be implemented by using the RFM model plus the translation transformation and affine transformation in image space based on RFM model transformation ${ }^{[16]}{ }^{[17]}$. In this paper, we use the transformation method in image space, namely, the correction of the image points is represented by affine transformation.

$$
\begin{aligned}
& x+\Delta x=x+a_{0}+a_{1} x+a_{2} y=R P C_{x}(\phi, \lambda, h) \\
& y+\Delta y=y+b_{0}+b_{1} x+b_{2} y=R P C_{y}(\phi, \lambda, h)
\end{aligned}
$$

Among them, $(x, y)$ donates the image coordinates, $(\varphi, \lambda, h)$ is ground coordinate of the image point, $\left(a_{0}, a_{1}, a_{2}\right)$ and $\left(b_{0}, b_{1}, b_{2}\right)$ are parameters of the affine transformation in image space, this is the first group of unknowns that need to solve in the RFM based block adjustment. Furthermore, the error equation of the adjustment model can be expressed as a matrix form:

$$
\begin{array}{lll}
v_{T P}=A_{T P} t+B_{T P} x-l_{T P} & P_{T P} \\
v_{G C P}= & B_{G C P} x-l_{G C P} & P_{G C P}
\end{array}
$$

In the formula, $B_{T P}$ and $B_{G C P}$ are the coefficient matrixes of ground coordinate correction of the tie point and the control point, respectively; $\boldsymbol{A}_{T P}$ is the coefficient matrix of affine transformation parameter correction; $\boldsymbol{l}_{\boldsymbol{T} P}$ and $\boldsymbol{l}_{\boldsymbol{G C P}}$ is the constant term matrix of the image point and the control point, respectively. $\boldsymbol{P}_{T P}, \boldsymbol{P}_{G C P}$ are weight matrixes of the tie point and the control point. $\boldsymbol{T}$ is the correction matrix of the affine transformation parameters in the image space, and $\boldsymbol{X}$ is the ground point coordinate correction matrix.

$$
\begin{aligned}
\boldsymbol{t} & =\left[\begin{array}{llllll}
\Delta a_{0} & \Delta a_{1} & \Delta a_{2} & \Delta b_{0} & \Delta b_{1} & \Delta b_{2}
\end{array}\right]^{T} \\
\boldsymbol{x} & =\left[\begin{array}{llll}
\Delta \varphi & \Delta \lambda & \Delta h
\end{array}\right]^{T}
\end{aligned}
$$

According to the least square principle, the corresponding normal equation matrix is:

$$
\left[\begin{array}{cc}
\boldsymbol{A}_{T P}^{T} \boldsymbol{P}_{T P} \boldsymbol{A}_{T P} & \boldsymbol{A}_{T P}^{T} \boldsymbol{P}_{T P} \boldsymbol{B}_{T P} \\
\boldsymbol{B}_{T P}^{T} \boldsymbol{P}_{T P} \boldsymbol{A}_{T P} & \boldsymbol{B}_{T P}^{T} \boldsymbol{P}_{T P} \boldsymbol{B}_{T P}+\boldsymbol{B}_{G C P}^{T} \boldsymbol{P}_{G C P} \boldsymbol{B}_{G C P}
\end{array}\right]\left[\begin{array}{l}
\boldsymbol{t} \\
\boldsymbol{x}
\end{array}\right]=\left[\begin{array}{c}
\boldsymbol{A}_{T P}^{T} \boldsymbol{P}_{T P} \boldsymbol{l}_{T P} \\
\boldsymbol{B}_{T P}^{T} \boldsymbol{P}_{T P} \boldsymbol{l}_{T P}+\boldsymbol{B}_{G C P}^{T} \boldsymbol{P}_{G C P} \boldsymbol{l}_{G C P}
\end{array}\right]
$$

On the actual solving process of block adjustment, due to the number of unknowns is too much, generally, a certain class of unknowns are eliminated first (general eliminating unknown $\mathrm{X})$ to obtain the reformation normal equation that only contains one class of unknowns $\boldsymbol{T}$, after solving the unknowns by using iterating block method, bringing it into error equations to calculate another class of unknowns. Generally, block adjustment is considered as an iteration process, each iteration solved the correction of unknowns and updated it, and then using it for the next iteration, the iteration would repeat until the preset convergence condition of the overall adjustment system is satisfied.

According to the imaging characteristics and acquisition methods of optical satellite images, block adjustment can be divided into stereo satellite images and single view images (under weak overlapping conditions) in the actual data processing. Stereo satellite image block adjustment is applied to the data processing of satellite stereo pairs (two or three views) and is mainly used for stereo mapping, DEM/DOM production, etc. Single view image (weak overlapping conditions) block adjustment, also known as planar block adjustment, is mainly used for the satellite image with small overlap area (small base height), this kind of satellite images often has week intersection angle $\left(\leq 10^{\circ}\right)$ of corresponding image rays, and difficult to build stereo image pairs for stereo mapping. for example, the mapping production of geographical national conditions monitoring project and second national land survey project selected this kind of single view images for block adjustment. For the planar block adjustment, using the stereo image block adjustment method directly will cause the problem of non-convergence of adjustment results caused by abnormal elevations of tie points. Therefore, the elevation value of the ground of the tie point is not calculated during the planar block adjustment, only the 
affine transformation parameters of the satellite image and the correction values of the ground plane coordinates $(\varphi, \lambda)$ are calculated, and the elevation $h$ is obtained by interpolation of the introduced DEM.

\section{OPTICAL SATELLITE IMAGE GCP INDEPENDENT BLOCK ADJUSTMENT MODEL}

\section{1 "Average" virtual control point based block} adjustment method without GCP for optical satellite image

Through the analysis of the residual systematic errors of optical satellite imagery and considering the problem which affects computation efficiency and stability of block adjustment, such as the huge amount of computation in block adjustment for large-scale imagery, low overlapping of local scope in actual data, short baseline, irregular block structure. In this paper, the Alternating Direction Method and total least squares adjustment methods are comprehensively used, we achieve the overall GCP-independent adjustment of the largescale satellite images in two steps.

The basic idea of "Alternating Direction Method [15]" is to decompose (convex optimization) global optimization problem into several pieces of smaller (parallel) problem that is easier to solve in local, and then through adjusting the solutions of these small problems to get the global solution of the problem. Firstly, we assumed that the affine transformation parameters $\left(a_{0}, a_{1}, a_{2}\right)$ and $\left(b_{0}, b_{1}, b_{2}\right)$ of each image in formulation (1) is given, and then the all the point coordinates $(\varphi, \lambda, h)$ in object space can be calculated, that is so-called "forward intersection", in this step, for each tie point, its dimension of normal equation is only a $3 \times 3$ matrix; Then we assumed that the coordinates $(\varphi, \lambda, h)$ of all points in object space is given, the affine transformation parameters $\left(a_{0}, a_{1}, a_{2}\right)$ and $\left(b_{0}, b_{1}, b_{2}\right)$ of each image can be calculated, that is so-called "resection", in this step, for each image, its dimension of normal equation is only a $6 \times 6$ matrix. Repeating the above two steps, until all the iteration changes of the unknowns' correction are less than a pre-set threshold. The advantage of alternating direction method is that it is suitable for parallel processing, because the solving of the affine transformation parameters of each image and the calculation of object space coordinates of each tie point are independent of each other, the disadvantage of it is the high number of iterations, but for this article, the initial value of all unknowns that need adjustment has meet the requirements. Differ from the method for generating virtual control points proposed in literature ${ }^{[8]}$, we use the method - GCPindependent adjustment method (GISIBA) proposed in this paper to generated the "average" virtual control points (figure 1 ), which is equivalent of using the "Alternating Direction Method" to build forward intersection for multiple stereo images, and getting the ground tie points $P_{1}, P_{2}, \ldots, P_{n}$ by it, then using the average point $\mathrm{P}$ of these ground tie point coordinates $P_{1}, P_{2}, \ldots, P_{n}$ to solve the pathological (rank loss) problem of the normal equation matrix encountered in solving GCP-independent adjustment. Due to the accuracy of direct ground positioning of different stereo images is different, so in the space forward intersection, the intersection point coordinates calculated by the space forward intersection from the same tie points in different stereo images is different, so we use the average ground coordinates of the tie point in all stereo images as the ground control point ("average" virtual control points), the precision of coordinate is improved by multiple iterations. On the one hand, it can use parallel computation to calculate the initial value of the unknown quickly, and on the other hand, the "good" initial value will be used to make the overall adjustment of the least square. Theoretically, GCP-independent adjustment method can make full use of a fact: "the randomness in residual systematic errors of images with the same coverage area, different time, different orbits and a long-time interval, the randomness even can be partially offset each other", thus, the positioning precision of satellite imagery can be improved after the overall GCP-independent adjustment.

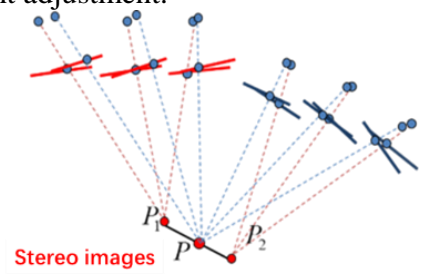

Figure 1: Principle of ground coordinate calculation of virtual "average" control points

For the tie point $k$ that appears on $n(n \geq 2)$ stereo images, with tie point $k$ passing through $i(i=1,2, \ldots, n)$ stereo image pairs, assume that $\left(\varphi_{k}^{i}, \lambda_{k}^{i}, h_{k}^{i}\right)$ is the ground coordinate calculated from orientation parameters of $i$ stereo image pairs by forward intersection, and $\left(\varphi_{k}, \lambda_{k}, h_{k}\right)$ is the average value of all $\left(\varphi_{k}^{i}, \lambda_{k}^{i}, h_{k}^{i}\right)$; thus, the average value of ground coordinates of tie points is:

$$
\left[\begin{array}{lll}
\varphi_{k} & \lambda_{k} & h_{k}
\end{array}\right]^{T}=\left[\begin{array}{ccc}
\sum_{i=1}^{n} \varphi_{k}^{i} & \frac{\sum_{i=1}^{n} \lambda_{k}^{i}}{n} & \frac{\sum_{i=1}^{n} h_{k}^{i}}{n}
\end{array}\right]^{T}
$$

Assume that $\left(\varphi_{k}^{o}, \lambda_{k}^{o}, h_{k}^{o}\right)$ is the true value of ground coordinates of the tie point, the coordinate error vector in object space is $\overrightarrow{\boldsymbol{r}_{\boldsymbol{k}}^{i}}$, which is calculated by the forward intersection with the orientation parameters of the ith stereo images of the tie point $k$ :

$$
\overrightarrow{\boldsymbol{r}_{\boldsymbol{k}}^{i}}=\left[\begin{array}{lll}
\varphi_{k}^{i}-\varphi_{k}^{o} & \lambda_{k}^{i}-\lambda_{k}^{o} & h_{k}^{i}-h_{k}^{o}
\end{array}\right]^{T}
$$

The error vector of average coordinate of the tie point in object space is $\overrightarrow{r_{k}}$ :

$$
\overrightarrow{\boldsymbol{r}_{\boldsymbol{k}}}=\left[\begin{array}{lll}
\varphi_{k}-\varphi_{k}^{o} & \lambda_{k}-\lambda_{k}^{o} & h_{k}-h_{k}^{o}
\end{array}\right]^{T}
$$

From (5) to (7):

$$
\overrightarrow{\boldsymbol{r}_{k}}=\frac{\sum_{i=1}^{n} \overrightarrow{\boldsymbol{r}_{k}^{i}}}{n}
$$

Assume that $\overrightarrow{\boldsymbol{r}_{k}^{\max }}$ is the maximum error of the tie points; according to the absolute value inequality, we can obtain:

$$
\left|\overrightarrow{\boldsymbol{r}_{k}}\right|=\left|\frac{\sum_{i=1}^{n} \overrightarrow{\boldsymbol{r}_{k}^{i}}}{n}\right| \leq \frac{\sum_{i=1}^{n} \overrightarrow{\boldsymbol{r}_{k}^{i}} \mid}{n} \leq\left|\overrightarrow{\boldsymbol{r}_{k}^{\max }}\right|
$$

By (9), it can be concluded that:

Conclusion-1: Assume that the initial GCP-independent positioning accuracy of the satellite imagery involved in processing is of the same order of magnitude (for example, approximately $50 \mathrm{~m}$ ); after using the "average" virtual control point to conduct the block adjustment, the final position accuracy of the image will be improved, and in the worst conditions, the result is still better than the worst initial 
positioning accuracy of the original image;

Conclusion-2: Assume that the initial GCP-independent positioning accuracy of the satellite imagery involved in processing is of the same order of magnitude; increasing the number of coverage image cannot always improve the overall positioning accuracy quickly, but in a statistical sense, the higher the number of the high-resolution satellite images covering the same area is, the more improved is the final positioning accuracy improved after the overall adjustment.

\subsection{GCP independent block adjustment model - GIBIBA}

The main steps of the process can be summarized as follows: (1) Assuming that affine transformation parameters of all images are given, according to the image space coordinates of tie point in different images, seeing the stereo image as the basic unit, we can solve the ground coordinates $(\varphi, \lambda, h)$ of the tie point in a stereo images by using forward intersection; If the tie point has multiple stereo images, the average value of the corresponding ground coordinate is taken as the ground coordinate of the tie point;

(2) Assuming that the ground coordinates of the tie points in each image are given, the affine transformation parameters of the image can be solved, in the process, at least six points are needed. This step has one equation involved for each image, which can be thought as a adjustment of a 6 x6 matrix, according to the unit weight root mean square error after adjustment, eliminating possible gross error point, in other words, eliminating the point the residual of which is greater than $3.0 \sim 5.0 \sigma_{0}$;

(3) Repeat steps (1) (2), until the image affine transformation parameters and correction of the ground coordinates of tie points are less than a certain value ( 0.2 pixels is selected in this article), and then the iteration ends.

(4) Based on the affine transformation parameters of the image, the ground coordinates and the image point coordinate residuals $\left(v_{x}, v_{y}\right)_{i, i=1, \ldots, n}$ are calculated for each tie point through the forward intersection. Where $\mathrm{n}$ is the "the number of rays" of each tie point, that is, the point appears in $\mathrm{n}$ images. Assuming $v_{i}=\operatorname{sqrt}\left(v^{2} x+v_{y}^{2}\right), \quad v_{\max }=\max \left(v_{i, i=1, \ldots, n}\right)$, for each tie point, we set the prior weight $p=1.0 / v^{2}$ max

(5) According to the ground coordinates and the corresponding prior weights of each tie points, on the basis of (1) to (4), a RFM based total least squares adjustment is carried out, and through the strategy of iterative choosing weights to eliminate the small scale gross error ${ }^{[15][22]}$.

From the above description, we can see that the core of the algorithm is through the "average" virtual control points to carry out the block adjustment without relying on the thirdparty geospatial data and ground control points, the purpose is making full use of the residual error randomness of the longtime series stereo satellite image covering the same imaging area to achieve high precision of the overall adjustment. Considering the generality of the algorithm, the ground coordinates of the control points will remain unchanged during the iteration, if there is a certain number of ground control points.

For single view satellite images with week overlapping, we need to introduce DEM of image area and used the method of planar block adjustment. So, step (1) and (4) need to change as followed in this situation:

$\left(1^{*}\right)$ Assuming that affine transformation parameters of all images are given, according to the image space coordinates of tie point in different images (more than 2 images), with the introduced DEM, we can solve the ground coordinates $(\varphi, \lambda$, $h$ ) of the tie point by using single ray intersection; If the tie point has multiple images, the average value of the corresponding plan ground coordinate is taken as the plan ground coordinate of the tie point $(\varphi, \lambda)$, and the elevation $h$ is obtained by interpolation of the introduced DEM.

(4*) Based on the affine transformation parameters of the image, the ground coordinates and the image point coordinate residuals $\left(v_{x}, v_{y}\right)_{i, i=1, \ldots, n}$ are calculated from ground point coordinates after step (3). Where $\mathrm{n}$ is the "the number of rays" of each tie point, that is, the point appears in $\mathrm{n}$ images. Assuming $v_{i}=\operatorname{sqrt}\left(v_{x}^{2}+v^{2}\right), \quad v_{\max }=\max \left(v_{i, i=1, \ldots, n}\right)$, for each tie point, we set the prior weight $p=1.0 / v^{2}$ max .

It can be seen from the above that the steps in block adjustment of the stereo images and planar images are basically the same. The joint block adjustment stereo images and planar images can be achieved by using step $\left(1^{*}, 2,3,4^{*}, 5\right)$ if elevation constraints (high-precision DEM or published 30m SRTM DEM) can be introduced.

\section{EXPERIMENT AND ANALYSIS}

To verify the adjustment precision of the proposed algorithm and the actual production performance as well as the applicability of the method in junction process of different overseas area, we selected four groups of worldwide ZY-3 stereo satellite image data and one ZY-3 and GF-1 single view satellite image data to verify the GISIBA overall GCPindependent adjustment algorithm regarding the following aspects: 1) the accuracy (experiments 1 and 2) and 2) the applicability (experiment 3 ) of the junction processing in actual production.

In this paper, based on the cluster computing environment built by our team (including 4 high performance computing nodes, with each compute node configured with an 8-core Intel Xeon E5-4650L CPU and 64 GB of memory and with the storage system having 2 SAS interfaces and a 500 GB hard disk system), the self-developed satellite image automatic matching method for tie point and GISIBA GCP-independent adjustment methods are used to process data (based on visual $\mathrm{c}++2015$ studio, 64bit OS)

The test scheme is as follows: first, use the tie points automatic matching method self-developed by our team to process the optical satellite images, and use a coarse-to-fine multi-level pyramidal image matching strategy for automatic extraction of tie points; next, based on extracted tie points, GISIBA block adjustment is used to obtain the high-precision image orientation parameters (including the affine transformation parameter corrections and the ground coordinate corrections of the tie points); finally, the accuracy and performance of our method are verified by different types of check points.

Table 1: description and scheme of different data sets for experiment

\begin{tabular}{|c|c|l|l|l|}
\hline $\begin{array}{c}\text { \#exp- } \\
\text { eriment }\end{array}$ & Data & \multicolumn{1}{c|}{ Information } & \multicolumn{1}{|c|}{ Accuracy Verification Scheme } & \multicolumn{1}{c|}{ Goal } \\
\hline 1 & Data 1-1 & $\begin{array}{l}\text { In China, there are 23 ZY-3 } \\
\text { images, covering nearly } \\
18,000 \mathrm{~km}^{2} \text {. The imaging }\end{array}$ & $\begin{array}{l}\text { Selecting 1300 uniform distributed } \\
\text { tie points, using the automatic } \\
\text { matching aided semi-automatic }\end{array}$ & $\begin{array}{l}\text { Comparing two sets of } \\
\text { adjustment results: 1) verify } \\
\text { the improvement of final }\end{array}$ \\
\hline
\end{tabular}




\begin{tabular}{|c|c|c|c|c|}
\hline & & $\begin{array}{l}\text { time is during } \\
2012.10 \sim 2013.04, \text { more than } \\
50 \% \text { areas are covered only } \\
\text { once in images. }\end{array}$ & $\begin{array}{l}\text { method, the ground coordinate is } \\
\text { measured and accuracy is verified } \\
\text { by the national basic mapping } \\
\text { 1:50000 scale DOM/DEM. }\end{array}$ & \multirow{2}{*}{$\begin{array}{l}\text { positioning accuracy of the } \\
\text { image using GISIBA method; } \\
\text { 2) verify the effect of } \\
\text { positioning accuracy after } \\
\text { overall adjustment by } \\
\text { increasing the coverage } \\
\text { number of images }\end{array}$} \\
\hline & Data 1-2 & $\begin{array}{l}\text { Outside China, } 1815 \mathrm{ZY}-3 \\
\text { images, covering nearly } \\
20,000 \mathrm{~km}^{2}, \text { imaging time is } \\
\text { during } 2012.02 \sim 2016.10, \\
\text { most of the areas are covered } \\
\text { about } 5 \text { times. }\end{array}$ & $\begin{array}{l}\text { By selecting } 2200 \text { uniform } \\
\text { distributed tie points, the automatic } \\
\text { matching aided semi-automatic } \\
\text { method is adopted to measure the } \\
\text { accuracy of ground coordinate with } \\
\text { a } 4.5 \mathrm{~m} \text { resolution Google DOM } \\
\text { /30m SRTM DEM. }\end{array}$ & \\
\hline 2 & Data 2 & $\begin{array}{l}\text { In China, there are } 1026 \mathrm{ZY}-3 \\
\text { images, covering about } \\
240,000 \mathrm{~km}^{2} . \text { The imaging } \\
\text { time is during } \\
2012.04 \sim 2016.02 \text {, and most } \\
\text { of the areas are covered by } \\
\text { three times. }\end{array}$ & $\begin{array}{l}191 \text { uniform GPS control points are } \\
\text { selected to verify the the absolute } \\
\text { accuracy. }\end{array}$ & $\begin{array}{l}\text { The reliability and accuracy } \\
\text { of GISIBA method are } \\
\text { verified by the measured } \\
\text { control points. }\end{array}$ \\
\hline 3 & Data 3 & $\begin{array}{l}\text { Outside China, overseas } 5241 \\
\text { views ZY-3 image, covering } \\
\text { about } 1.4 \text { million } \mathrm{km}^{2} \text {, } \\
\text { imaging time } \\
2012.02 \sim 2017.04,70 \% \text { of the } \\
\text { area has been covered about } 3 \\
\text { times, and the eastern image } \\
\text { coverage is less. }\end{array}$ & $\begin{array}{l}\text { Test area is divided into east and } \\
\text { west two partitions, } 3 \text { rails of the } \\
\text { data have overlap between the two } \\
\text { areas, selecting } 1100 \text { uniformly } \\
\text { distributed tie points, using the } \\
\text { automatic matching aided semi- } \\
\text { automatic method, using Google } \\
\text { DOM / } 30 \text { m SRTM DEM with } 4.5 \\
\text { m resolution to measure ground } \\
\text { coordinates and verify the accuracy }\end{array}$ & $\begin{array}{l}\text { Based on the comparison and } \\
\text { analysis of the results of the } \\
\text { three groups in the eastern, } \\
\text { western and overall groups, } \\
\text { the strategy and advantages of } \\
\text { using GISIBA method to } \\
\text { solve the mosaic problem are } \\
\text { proposed. }\end{array}$ \\
\hline 4 & Data 4 & $\begin{array}{l}\text { In China, } 1766 \text { views of } \mathrm{ZY}- \\
32.1 \mathrm{~m} \text { resolution down view } \\
\text { images and } 2598 \text { views of } \\
\text { GF1 PMS1/2 } 2 \mathrm{~m} \text { resolution } \\
\text { images, covering about } \\
800,000 \mathrm{~km}^{2} \text {, imaging time } \\
2015.01 \sim 2016.08 \\
\end{array}$ & $\begin{array}{l}\text { Geometric constraints of horizontal } \\
\text { is } 15 \mathrm{~m} \text { ETM+ DOM and vertical is } \\
30 \mathrm{~m} \text { ASTER DEM }\end{array}$ & $\begin{array}{l}\text { verify DOM product accuracy } \\
\text { and productivity for large } \\
\text { area. }\end{array}$ \\
\hline
\end{tabular}

\subsection{Experiment 1: GCP-independent adjustment experiment of a stereo image with different degrees and multiple phases of coverage with $\mathrm{ZY}-3$}

Data1-1 contains 23 ZY-3 satellite images (8 three linear array and stereo images, table 1 ), over $50 \%$ of the areas are only covered once, and the positioning error of the original data without ground control is up to $29.0 \mathrm{~m}$. In the data processing, 29661 tie points were extracted, and the unit weight root mean square error (RMSE) $\sigma_{0}$ after GISIBA overall adjustment was 0.34 pixels. The plane error distribution of 1300 checkpoints is shown in figure 2 , and the accuracy evaluation results are shown in table 2 .

Data 1-2: 1815 ZY-3 satellite images (505 three linear array and stereo images, table 1), with most of the areas covered 5 times by three linear array and stereo images; the positioning error of the original data without GCPs is up to $24.9 \mathrm{~m}$. A total of 903,244 tie points were extracted in the data processing, and the unit weight RMSE $\sigma_{0}$ after the overall adjustment was 0.37 pixels. The plane error distribution of 2200 checkpoints is shown in figure 3 , and the accuracy evaluation results are shown in table 2 .
The results of the two groups indicate that the following: 1) The unit weight RMSE after GISIBA-based overall adjustment proposed in this paper is 0.37 pixels, with the accuracy increased from $29 \mathrm{~m}$ of the original data to better than $15 \mathrm{~m}$ for plane accuracy; thus, the proposed approach can significantly improve the positioning accuracy of overall adjustment of large scale satellite image without GCPs (verified Conclusion-1).

2) The precision of adjustment is improved by adding the degree of image coverage: in the experiment of data 1-2, the time span of the data is 5 years, the degree of coverage of the satellite image in the test area is 5, the GCP-independent adjustment using the data can achieve precision better than plane precision of $6.0 \mathrm{~m}$ and height accuracy of $2 \mathrm{~m}$. From the distribution of error vector in figure 3 (the scale of the vector in figure 3 is only 0.1 of figure 2), we can also see that adjustment results basically are unbiased. In contrast to the result of data 1-2, data 1-1 has less time span - only 1 year and lower degree of coverage, and its GCP-independent adjustment result has obvious systematic error, although it also achieved precision better than the plane precision $15 \mathrm{~m}$ (verified Conclusion-2).

Table 2: Accuracy comparison between two groups of test data (heights have been transformed from geodetic heights to normal heights)

\begin{tabular}{|c|c|c|c|c|c|c|}
\hline & \multicolumn{3}{|c|}{ Mean square error (m) } & \multicolumn{4}{c|}{ average value (m) } \\
\cline { 2 - 7 } & $\mathrm{Sx}$ & sy & sz & ax & ay & az \\
\hline Date 1-1 & 13.90 & 6.38 & 3.14 & 12.89 & 6.00 & 3.14 \\
\hline Date 1-2 & 2.36 & 5.98 & 2.07 & -1.50 & -3.97 & 1.37 \\
\hline
\end{tabular}




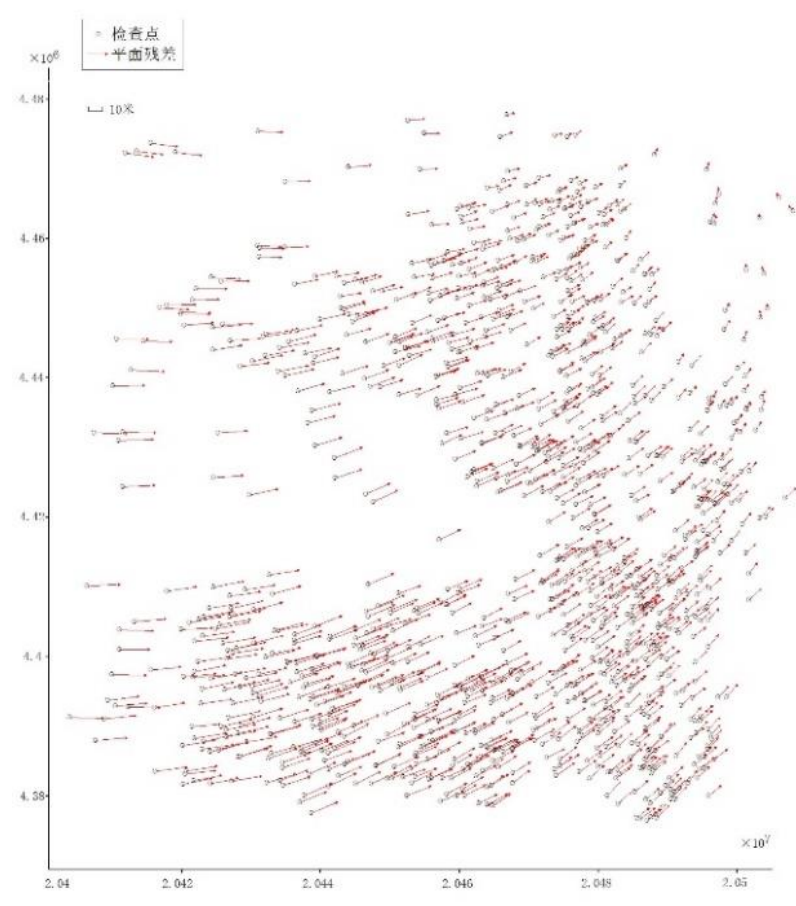

Fig 2: Test data 1: horizontal error distribution from 1300 check points for $23 \mathrm{ZY}-3$ satellite images ( 8 three-line array and stereo images) (the scale of the error vector is $10 \mathrm{~m}$ )

\subsection{Experiment 2: Verification of the absolute accuracy of}

\section{GCP-independent block adjustment in a stereo image with} ZY-3

The experimental area includes the southern part of Yunnan province and the western region of Guangxi province, and the data includes 1026 "ZY-3" satellite images (264 three linear array and stereo images, table 1). During data processing, a stereo image was deleted (most of the areas are covered by clouds), 2581578 points were extracted, and 1574435 points remained after automatically rejecting gross error; the adjustment calculation required less than four minutes. In the experiment, we used 191 uniformly distributed GPS measurement control points to conduct the precision analysis of GISIBA-based GCP-independent adjustment (figure 4); the accuracy analysis results are shown in table 3 .

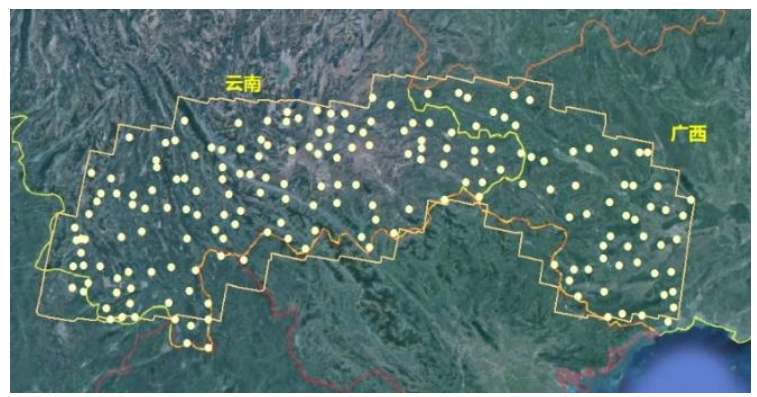

Fig 4: Distribution of GCPs in the Yunnan and Guangxi regions

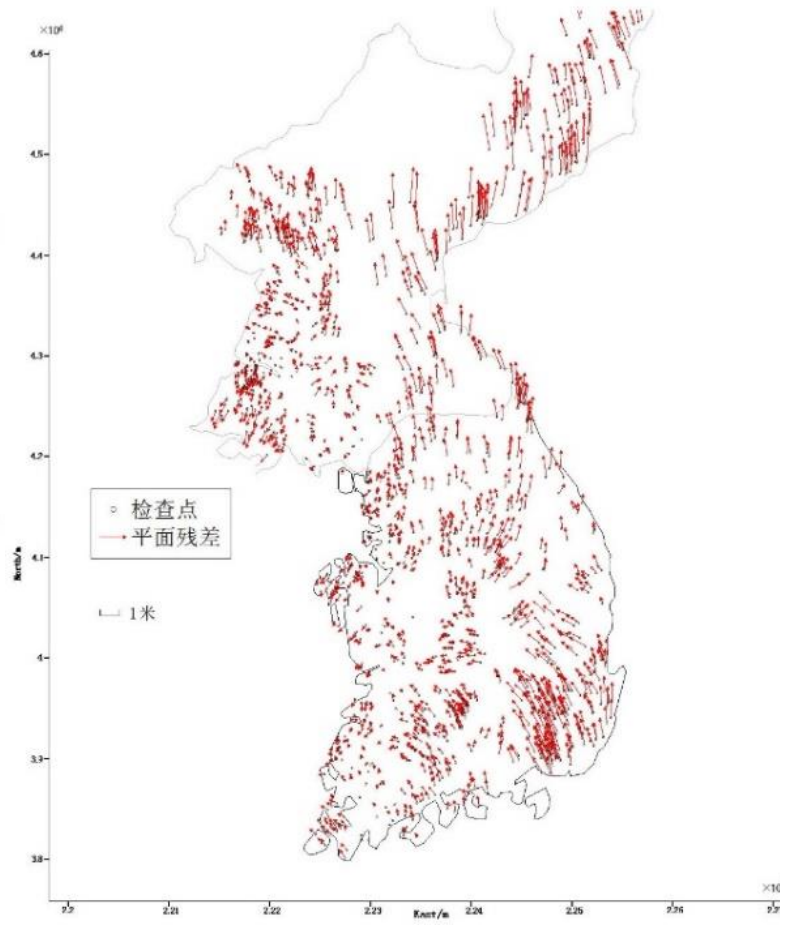

Fig 3: Test data 2: horizontal error distribution from 2200 check points for $1815 \mathrm{ZY}-3$ satellite images (605 three-line array and stereo images) (the scale of the error vector is 1

m)

Table 3: Adjustment accuracy analysis with 191 GCPs in the Yunnan and Guangxi regions

(heights have been transformed from geodetic heights to normal heights)

\begin{tabular}{|c|c|c|c|c|c|c|}
\hline \multirow{2}{*}{} & \multicolumn{3}{|c|}{ Mean square error (m) } & \multicolumn{3}{c|}{ average value (m) } \\
\cline { 2 - 7 } & sx & sy & sz & ax & ay & az \\
\hline Data 2 & 4.36 & 5.35 & 4.82 & 3.34 & 4.40 & 2.85 \\
\hline
\end{tabular}

The accuracy analysis results of the measured control points of control showed that, after accuracy examination of 191 measured control points, using ZY-3 satellite image with for nearly five years of time span and 3 degrees of coverage to conduct overall GCP-independent adjustment can achieve precision better than plane precision of $6.0 \mathrm{~m}$ and elevation accuracy of $5.0 \mathrm{~m}$. The precision of the GISIBA-based GCPindependent adjustment method is fully verified.

\subsection{Experiment 3: GCP-independent block adjustment and block mosaic test with $\mathrm{ZY}-3$}

The experimental area contains 5241 ZY-3 satellite images (1382 three linear array and stereo image pairs, table 1). The maximum positioning error of the original data without ground control points is $29.7 \mathrm{~m}$ (there are two abnormal images, for which the error is greater than 100 pixels). To illustrate the problem of block mosaic, we divide the test data into east and west partitions; the eastern partition contains 653 stereo images, the western partition contains 808 stereo images, and there are overlaps on three orbits of images between two partitions. The precision analysis result using 1100 check points is shown in table 4 : 
(heights have been transformed from geodetic heights to normal heights)

\begin{tabular}{|c|c|c|c|c|c|c|c|}
\hline & Unit weight RMSE & \multicolumn{3}{|c|}{ Mean square error $(\mathrm{m})$} & \multicolumn{3}{|c|}{ average value $(\mathrm{m})$} \\
\cline { 3 - 8 } & $(\mathrm{px})$ & $\mathrm{sx}$ & $\mathrm{sy}$ & $\mathrm{sz}$ & $\mathrm{ax}$ & $\mathrm{ay}$ & $\mathrm{az}$ \\
\hline East partition & 0.42 & 5.77 & 4.47 & 13.12 & 0.59 & -1.25 & -10.12 \\
\hline West partition & 0.43 & 3.00 & 2.51 & 6.71 & 1.07 & -1.12 & 0.98 \\
\hline The whole area & 0.39 & 4.62 & 3.74 & 5.22 & 1.11 & -1.41 & -4.25 \\
\hline
\end{tabular}

The results show that:

1) Because of the use of ZY-3 satellite images having 6 years of time span and multiple coverage in the test area, the overall adjustment without ground control points achieved the plane precision of better than $6.0 \mathrm{~m}$ in the east and west partitions, without obvious systematic error.

2) 49 hours is required to automatically extract more than 10 million tie points during the overall GCP-independent block adjustment processing; the block adjustment steps requires only 7.5 minutes, the plane precision without GCPs of the whole area is better than $5.0 \mathrm{~m}$ after the adjustment, with no obvious systematic error, and the elevation RMSE reached 6.0 m.

Considering the applicability of the algorithm, it is very difficult to conduct the mosaic processing of the two partitions in the actual production, especially for elevation mosaic processing. To solve the mosaic problem of adjustment result of the eastern and western partitions, based on the verification of the calculating speed and stability of the GISIBA-based GCP-independent adjustment methods, Google DOM with 4.5 $\mathrm{m}$ resolution and SRTM DEM with $30 \mathrm{~m}$ resolution are introduced as the geometric constraints in plane and elevation precision, and part of the uniform distributed tie points will be registered with the data having geospatial information by automatic matching algorithm, followed by interpolating the corresponding ground coordinates. Next, in the process of GISIBA-based overall adjustment, these tie points will be introduced in the adjustment as the observed value of weighted "reference point", with its weight decided by the plane of 50 $\mathrm{m}$ and the elevation of $20 \mathrm{~m}$. We still evaluate the accuracy using 1100 checkpoints selected in the whole test area; the results of the block adjustment after introducing these geometric constraints is shown below (table 5).

Table 5: Adjustment accuracy comparison of the east, west and whole teat areas with horizontal and vertical geometric constraints (heights have been transformed from geodetic heights to normal heights)

\begin{tabular}{|c|c|c|c|c|c|c|c|}
\hline & Unit Weight RMSE (px) & \multicolumn{2}{|c|}{ Mean square error $(\mathrm{m})$} & \multicolumn{3}{|c|}{ average value $(\mathrm{m})$} \\
\cline { 3 - 8 } & & $\mathrm{sx}$ & $\mathrm{sy}$ & $\mathrm{sz}$ & $\mathrm{ax}$ & ay & az \\
\hline East partition & 0.41 & 0.86 & 0.84 & 2.07 & 0.01 & -0.00 & 0.00 \\
\hline West partition & 0.43 & 1.02 & 0.96 & 2.36 & 0.00 & 0.00 & 0.02 \\
\hline The whole area & 0.40 & 0.79 & 0.80 & 2.08 & 0.00 & 0.00 & 0.00 \\
\hline
\end{tabular}

The experimental results show that, after introducing geometric constraints of plane and elevation, compared with the third-party geographic information data, the east and west partitions of the whole area achieved a plane precision better than $1 \mathrm{~m}$, an elevation precision better than $3 \mathrm{~m}$, and exhibited no systematic error; thus, this method solved mosaic problem of the adjustment result of east and west area.

An aspect to consider in the actual production is that, after introducing the plane/vertical geometric constraints, the overall block adjustment result shows a well "fitting" accuracy of the reference data, although the obvious error of reference data will be eliminated as the gross error in the process of adjustment; nevertheless, we still recommend the use third- party geographic information data with given and verifiable precision as the plane and vertical geometric constraints.

\subsection{Experiment 4: Stereo and single view satellite images block adjustment with ZY-3 and GF-1}

The testing area has 1766 views of ZY-3 2.1m resolution down view images and 2598 views of GF-1 PMS1/2 2m resolution images (Figure 5), covering about $800,000 \mathrm{~km}^{2}$. This set of experimental data is a typical single view image data with weak overlapping condition, and is mainly used to meet the DOM production with a resolution of $2 \mathrm{~m}$.

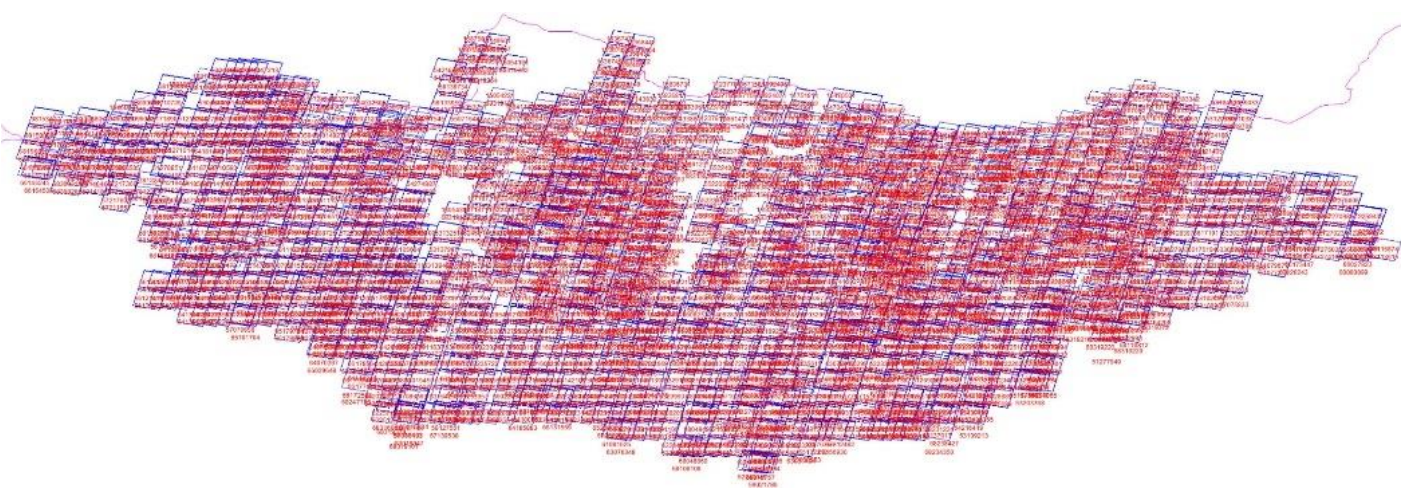

Figure 5: Overview of ZY-3 and GF-1 satellite image distributions 
Considering the actual production requirements, the geometric constraints of horizontal is $15 \mathrm{~m} \mathrm{ETM+}$ DOM and vertical is $30 \mathrm{~m}$ ASTER DEM used in this experiment. 5.73million tie points were extracted after 19.2 hours image matching processing, and 380,000 reference points were automatic matched from geometric constraints of horizontal and vertical. The unit weight root mean square error after GISIBA overall adjustment was 0.35 pixels.

As a key indicator for the DOM production of using satellite imagery, the DOM mosaic accuracy

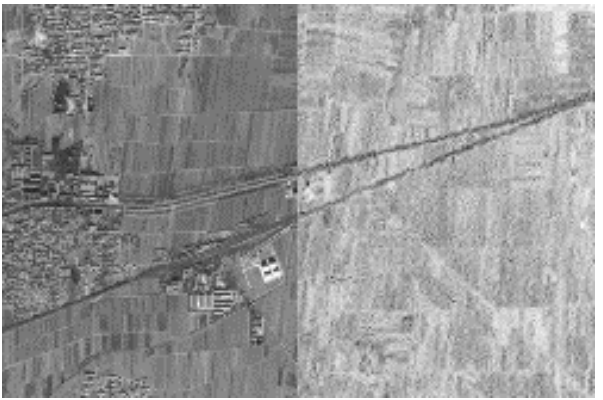

Figure 6: The sketch map of mosaic DOM

\section{CONCLUSIONS}

Aiming at the features of imaging geometry of domestic optical high-resolution satellite images and the problem existed in GCP-independent precise positioning technology, based on the "Alternate Direction Method" and RFM-based least square overall block adjustment, we proposed a GCP-Independent Block Adjustment method- GISIBA.

We use multiple sets of "ZY-3" "GF-1" satellite image data of the typical regions to verify our method, the experiment results show that: 1) due to the use of "Alternate Direction Method" and RFM-based least square overall block adjustment, this method can make full use of both of their advantages: low dependence on initial value, high computing speed, uniform distributed accuracy, and the method is stable and reliable enough. 2) in this method, the "average" virtual control points are built, though the points, on the one hand, the "rank" problem led by non-convergence in GCP-independent block adjustment can be solved, on the other hand, our method can make full use of the randomness of residual systematic errors of the stereo satellite images having the same coverage area in a long time sequence to carry out the GCP-independent block adjustment, without relying on the third-party geospatial data and ground control points, and can achieve the plane precision better than $6.0 \mathrm{~m}$ and elevation precision better than $5 \mathrm{~m}$. 3) the method has high generality, by introducing the third-party geographic information data with given and verifiable precision as the plane and vertical geometric constraint (weak auxiliary control), the method can mosaic problem of the adjustment results in different areas effectively, which is always encountered in the actual production.

The software system was applied and improved in the 2016 and 2017 global mapping trial production, providing technical support for the global geographic information resource construction project and the characterizes the consistency of the geometric positioning accuracy between same features of adjacent DOM. Therefore, corresponding points in the multi-images were used for accuracy checking of DOM mosaic (Figure 6), the root mean square error of mosaic is 0.41 pixels, and the maximum error is 2.1 pixels. This set of experimental data validates the efficiency and accuracy of our algorithm in the DOM production of large area.

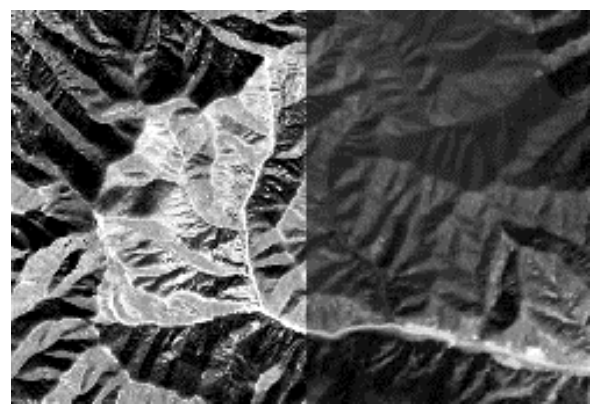

(left: plan area; right: mountain area)

production of domestic optical satellite imagery highprecision mapping products.

\section{REFERENCE}

[1] LI Deren, WANG Mi. On-orbit Geometric Calibration and Accuracy Assessment of ZY-3 [J]. Spacecraft Recovery \& Remote Sensing, 2012, 33(3):1-6. DOI : $\quad 10.3969 /$ j.issn.10098518.2012.03.001.

[2] TANG Xinming, WANG Hongyan, ZHU Xiaoyong. Technology and Applications of Surveying and Mapping for ZY-3 Satellites [J]. Acta Geodaetica et Cartographica Sinica, 2017, 46 (10): 1482-1491. DOI: 10.11947/ j.AGCS.2017.20170251.

[3] WANG Renxiang, HU Xin, WANG Jianrong. Photogrammetry of Mapping Satellite without Ground Control Points [J]. Acta Geodaetica et Cartographica Sinica, 2013, 42(1):1-5.

[4] WANG Renxiang, WANG Jianrong. Discussion on satellite photogrammetry without ground control point $[\mathrm{J}]$. Science of Surveying and Mapping, 2015, 40(2): 3-12. DOI: $10.16251 /$ j.cnki.1009 2307.2015.02.001.

[5] WANG Renxiang, WANG Jianrong, HU Xin. Preliminary Location Accuracy Assessments of 3rd Satellite of TH-1[J]. Acta Geodaetica et Cartographica Sinica, 2016, 45(10):1135-1139. DOI:10.11947/ j.AGCS.2016.20160373.)

[6] GONG Jianya, WANG Mi, YANG Bo. Highprecision Geometric Processing Theory and Method of High -resolution Optical Remote Sensing Satellite Imagery without GCP [J]. Acta Geodaetica et Cartographica Sinica, 2017, 46(10):1255G1261.

DOI: 10.11947/j.AGCS.2017.20170307.

[7] WANG Mi, YANG Bo, LI Deren, GONG Jianya, 
et al. Technologies and Applications of Block Adjustment Without Control for ZY-3 Images Covering China $[\mathrm{J}]$. Geomatics and Information Science of Wuhan University, 2017, 42(4): 427433. DOI:10.13203/ j.whugis20160534.

[8] YANG Bo, WANG Mi, PI Yingdong. Block adjustment without GCPs for Large scale Regions only Based on the Virtual Control Points[J]. Acta Geodaetica et Cartographica Sinica, 2017, 46(7): 874-881. DOI:10.11947/ j.AGCS.2017.20160588.

[9] CHEN Yuanyuan, TANG Zhiqiang, Wu Fang. Research of Surveying and Mapping Methods Without Control Based on "Google Earth + SAR" Images $[\mathrm{J}]$. Geomatics Technology and Equipment, 2015, 4(17): 17-19.

[10] LIU Chubin, ZHANG Yongsheng, FAN Dazhao, LEI Rong, et al. Research on the Geometrical Positioning Evaluation of ZY-3 Satellite at Abroad [J]. Bulletin of Surveying and Mapping, 2015(9):6-8 DOI:10.13474/j.cnki.112246.015.0266.

[11] LIU Chubin, ZHANG Yongsheng, TIAI Ye, FAN Dazhao, LEI Rong. First results of geometrical Positioning Evaluation of TH-1 satellite at abroad[J]. Journal of Liaoning Technical University (Natural Science), 2016, 35(6):657$660 . \quad$ DOI: $\quad 10.11956 /$ j.issn. $1008-$ 0562.2016.06.019.

[12] ZHANG Hao, ZHANG Guo, JIANG Yonghua, et al. A SRTM-DEM-controlled Ortho-rectification Method for Optical Satellite Remote Sensing Stereo Images[J]. Acta Geodaetica et Cartographica Sinica, 2016, 45(3):326-331. DOI:10.11947/ j.AGCS. 2016.20150358.

[13] CHEN Xiaowei, ZHANG Baoming, ZHANG Tonggang, et al. Public DEM-assisted Positioning Method for Chinese Satellite Imagery without Ground Control Points [J]. Acta Geodaetica et Cartographica Sinica, 2016, 45(11):1361-1370. DOI:10.11947/ j.AGCS.2016.20160317.

[14] WANG Taoyang, ZHANG Guo, LI Deren, et al. Comparison between Plane and Stereo Block
Adjustment for ZY-3 Satellite Images[J]. Acta Geodaetica et Cartographica Sinica, 2014, 43(4):389-395. DOI: $10.13485 /$ j.cnki.112089.2014.0058.

[15] Wang Zhizhuo. Principle of Photogrammetry [M]. Wuhan: Wuhan University Press, 2007, ISBN 978-7-307-05479-0, pp:138-139.

[16] Dial Gene., Jacek Grodecki.. IKONOS Stereo Accuracy Without Ground Control [A]. Proceedings of ASPRS 2003 Conference, Anchorage Alaska, May 5-9.

[17] ZHANG Li, ZHANG Jixian, CHEN Xiangyang, AN Hong. Block-Adjustment with SPOT-5 Imagery and Sparse GCPs Based on RFM [J]. Acta Geodaetica et Cartographica Sinica, 2009, 38(4): 302-310

[18] ZHANG Li, AI Haibin, XU Biao, et al. Automatic Tie-point Extraction Based on Multiple-image Matching and Bundle Adjustment of Large Block of Oblique Aerial Images[J]. Acta Geodaetica et Cartographica Sinica, 2017, 46(5): 554-564. DOI: 10.11947/j.AGCS.2017.20160571.

[19] ZHANG Zuxun, TAO Pengjie. An Overview on "Cloud Control" Photogrammetry in Big Data Era [J]. Acta Geodaetica et CartographicaSinica, 2017, 46(10):1238-1248.

DOI: 10.11947/j.AGCS.2017.20170337.

[20] Zhang Zuxun, Lu Lupin, Tao Pengjie. Registration of CBERS-02B Satellite Imagery in quick GIS Updating [A], In: SPIE proceedings of the $7 \mathrm{rd}$ SPIE international symposium multispectral image processing and pattern recognition, 2011, Guilin.

[21] Stephen Boyd, Neal Parikh, Eric Chu, et al. Distributed Optimization and Statistical Learning via the Alternating Direction Method of Multipliers [J]. Foundations and Trends in Machine Learning. 2010, 3(1): 1-122, DOI: $10.1561 / 2200000016$.

[22] Li Deren. Error Handling and Reliability Theory

[M]. Beijing: Surveying Press, 1988. 